\title{
Ausgewählte Studienliteratur
}

BaltL, Hermann u. Kocher, Gernot: Österreichische Rechtsgeschichte, ${ }^{8} 1995$. Berman, Harold J.: Recht und Revolution. Die Bildung der westlichen Rechtstradition, 1991 = stw 1203, 1995.

BOCKENFÖRDE, Ernst-Wolfgang (Hg.): Moderne deutsche Verfassungsgeschichte (1815-1914), ${ }^{2} 1981$.

BoLDT, Hans: Deutsche Verfassungsgeschichte, 2 Bde., ${ }^{2} 1990,1990$.

Botzenhart, Manfred: Deutsche Verfassungsgeschichte 1806-1949, 1993.

Brauneder, Wilhelm u. LACHMAYER, Friedrich: Österreichische Verfassungsgeschichte. Einführung in Entwicklung und Strukturen, ${ }^{6} 1992$.

Brunner, Otto, Conze, Werner u. Koselleck, Reinhart (Hgg.): Geschichtliche Grundbegriffe. Historisches Lexikon zur politisch-sozialen Sprache in Deutschland, 7 Bde., 1972-1992, Registerbd. in Vorbereitung.

CARLEN, Louis: Rechtsgeschichte der Schweiz. Eine Einführung, ${ }^{3} 1988$.

CoIng, Helmut: Europäisches Privatrecht, Bd. I: Älteres Gemeines Recht (1500-1800), 1985, Bd. II: 19. Jahrhundert: Überblick über die Entwicklung des Privatrechts in den ehemals gemeinrechtlichen Ländern, 1989.

CoING, Helmut ( $\mathrm{Hg}$.): Handbuch der Quellen und Literatur der neueren europäischen Privatrechtsgeschichte, 3 Bde. in 8 Teilbdn., 1973-1988.

ConRad, Hermann: Deutsche Rechtsgeschichte, 2 Bde., ${ }^{2} 1962,1966$.

DILCHER, Gerhard: Der rechtsgeschichtliche Grundlagenschein, 1979.

Dilcher, Gerhard u. HoRN, Norbert (Hgg.): Sozialwissenschaften im Studium des Rechts, Bd. IV: Rechtsgeschichte, 1978.

Döhring, Erich: Geschichte der deutschen Rechtspflege seit 1500, 1953.

Duchнard, Heinz: Deutsche Verfassungsgeschichte 1495-1806, $1991=$ Urban Tb. 417.

DURIG, Günther u. Rudolf, Walter (Hgg.): Texte zur deutschen Verfassungsgeschichte, ${ }^{3} 1996$.

Ebel, Friedrich u. Thielmann, Georg: Rechtsgeschichte, Bd. 1: Antike und Mittelalter, 1989; EBEL, Friedrich: Rechtsgeschichte, Bd. 2: Neuzeit, 1993.

EbeL, Wilhelm: Geschichte der Gesetzgebung in Deutschland, Neudr. d. 2. Aufl., 1988.

Eisenhardt, Ulrich: Deutsche Rechtsgeschichte, ${ }^{2} 1995$.

ERDO, Péter: Introductio in Historiam Scientiae Canonicae, Roma 1990.

Erler, Adalbert, Kaufmann, Ekkehard u. Werkmuller, Dieter (Hgg.): Handwörterbuch zur deutschen Rechtsgeschichte (HRG), Bd. 1: Aachen - Haussuchung, 1971, Bd. 2: Haustür - Lippe, 1978, Bd. 3: List - Politische Klausel, 1983, Bd. 4: Protonotar - Strafprozeßordnung, 1990, Bd. 5 im Erscheinen.

FensKe, Hans: Deutsche Verfassungsgeschichte, ${ }^{3} 1991$.

FlossmanN, Ursula: Österreichische Privatrechtsgeschichte, ${ }^{2} 1992$. 
Ausgewählte Studienliteratur

Forschungen zur Rechtsarchäologie und Rechtlichen Volkskunde, $1978 \mathrm{ff}$.

Gebhardt, Bruno u. Grundmann, Herbert (Hgg.): Handbuch der deutschen Geschichte, 4 Bde., ${ }^{9} 1970-{ }^{9} 1976$.

Gierke, Otto von: Deutsches Privatrecht, Bd. 1: Allgemeiner Teil und Personenrecht, 1895, Bd. 2: Sachenrecht, 1905, Bd. 3: Schuldrecht, 1917.

GILISSEN, John (Hg.): Bibliographische Einführung in die Rechtsgeschichte und Rechtsethnologie, D/2: Deutschland, bearb. v. Hans ThIEME, Wolfgang LeISER u. Bernhard Diestelkamp, 1970.

GMU'R, Rudolf: Grundriß der deutschen Rechtsgeschichte, ${ }^{7} 1996$.

GREWE, Wilhelm G.: Epochen der Völkerrechtsgeschichte, ${ }^{2} 1988$.

Grimm, Dieter: Deutsche Verfassungsgeschichte 1776-1866. Vom Beginn des modernen Verfassungsstaates bis zur Auflösung des Deutschen Bundes, 1988 = edition suhrkamp 1271 .

Hatrenhauer, Hans: Die deutschrechtliche Exegese. Eine Anleitung für Studenten, 1975.

Hattenhauer, Hans: Europäische Rechtsgeschichte, ${ }^{2} 1994$.

Hattenhauer, Hans: Die geistesgeschichtlichen Grundlagen des deutschen Rechts, ${ }^{4} 1996=$ UTB 1042.

Hattenhauer, Hans u. Buschmann, Arno (Hgg.): Textbuch zur Privatrechtsgeschichte der Neuzeit mit Übersetzungen, 1967.

Hintze, Otto: Staat und Verfassung. Gesammelte Abhandlungen zur allgemeinen Verfassungsgeschichte, hg. v. Gerhard Oestreich, ${ }^{3} 1970$.

Historische Zeitschrift (HZ), 1859-1943, $1949 \mathrm{ff}$.

Hofmann, Hanns Hubert: Quellen zum Verfassungsorganismus des Heiligen Römischen Reiches Deutscher Nation 1495-1813, 1976.

HOKE, Rudolf: Österreichische und deutsche Rechtsgeschichte, ${ }^{4} 1992$.

HOKE, Rudolf u. ReITER, Ilse (Hgg.): Quellensammlung zur österreichischen und deutschen Rechtsgeschichte vornehmlich für den Studiengebrauch, 1993.

Huber, Ernst Rudolf: Deutsche Verfassungsgeschichte seit 1789, 8 Bde., ${ }^{2} 1990$ (Revid. Nachdr. 1995)-1991.

HuBER, Ernst Rudolf (Hg.): Dokumente zur deutschen Verfassungsgeschichte, 4 Bde., ${ }^{3} 1978-^{3} 1992$, Registerbd. in Vorbereitung.

Huber, Ernst Rudolf u. Huber, Wolfgang (Hgg.): Staat und Kirche im 19. und 20. Jahrhundert. Dokumente zur Geschichte des deutschen Staatskirchenrechts, 4 Bde., 1973-1988.

Ius Commune. Zeitschrift für europäische Rechtsgeschichte, $1967 \mathrm{ff}$.

Jeserich, Kurt G. A., Pohr, Hans u. UnRuh, Georg-Christoph von (Hgg.): Deutsche Verwaltungsgeschichte, 6 Bde., 1983-1988.

JoAChimsen, Paul: Der deutsche Staatsgedanke von seinen Anfängen bis auf Leibniz und Friedrich den Großen. Dokumente und Entwicklung, 1921 (Nachdr. 1967).

KASPERS, Heinrich: Vom Sachsenspiegel zum Code Napoléon. Kleine Rechtsgeschichte im Spiegel alter Rechtsbücher, ${ }^{3} 1972$.

Kaufmann, Ekkehard: Deutsches Recht, 1984.

KERN, Eduard: Geschichte des Gerichtsverfassungsrechts, 1954.

Kimminich, Otto: Deutsche Verfassungsgeschichte, ${ }^{2} 1987$. 
Ausgewählte Studienliteratur

Kleinheyer, Gerd u. SCHROdER, Jan (Hgg.): Deutsche Juristen aus fünf Jahrhunderten. Eine biographische Einführung in die Geschichte der Rechtswissenschaft, ${ }^{3} 1989$ = UTB 578.

KÖвLER, Gerhard: Bilder aus der deutschen Rechtsgeschichte von den Anfängen bis zur Gegenwart, 1988.

KOBLER, Gerhard: Einfache Bibliographie europäisch-deutscher Rechtsgeschichte, 1990.

KOBLER, Gerhard: Deutsche Rechtsgeschichte, ${ }^{5} 1996$.

KOCHER, Gernot: Zeichen und Symbole des Rechts. Eine historische Ikonographie, 1992.

Koschaker, Paul: Europa und das römische Recht, ${ }^{4} 1966$.

Kroeschell, Karl: Deutsche Rechtsgeschichte 1 (bis 1250), ${ }^{10} 1992,2$ (1250-1650), ${ }^{8} 1992,3$ (seit 1650), ${ }^{2} 1993$ = WV-Studium Bde. 8, 9, 139.

Kroeschell, Karl: Rechtsgeschichte Deutschlands im 20. Jahrhundert, $1992=$ UTB 1681 .

KROGER, Klaus: Einführung in die jüngere deutsche Verfassungsgeschichte (1806-1933), 1988.

KunKel, Wolfgang u. a. (Hgg.): Quellen zur Neueren Privatrechtsgeschichte Deutschlands, 2 Bde. in 4 Halbbdn., 1936-1969.

Leuschner, Joachim (Hg.): Deutsche Geschichte, 10 Bde., ${ }^{3} 1988-^{2} 1982=\mathrm{Kl}$. Vandenhoeck-Reihe.

Lexikon des Mittelalters (LexMA), Bde. I (1980), II (1983), III (1986), IV (1989), V (1991), VI (1993), VII (1995), Bd. VIII im Erscheinen.

LUTGE, Friedrich: Deutsche Sozial- und Wirtschaftsgeschichte. Ein Überblick, ${ }^{3} 1966$ (Nachdr. 1979).

Maier, Hans, Rausch, Horst u. Denzer, Horst: Klassiker des politischen Denkens, Bd. I, ${ }^{6} 1986$; Bd. II, ${ }^{5} 1987$.

MAISEL, Witold: Rechtsarchäologie Europas, 1992.

Mann, Golo: Deutsche Geschichte des 19. und 20. Jahrhunderts, Ausg. 1992 = Fischer TB 11330.

MARCic, René: Geschichte der Rechtsphilosophie. Schwerpunkte - Kontrapunkte, 1971.

Menger, Christian-Friedrich: Deutsche Verfassungsgeschichte der Neuzeit. Eine Einführung in die Grundlagen, ${ }^{8} 1993=$ UTB 930.

MrtTeis, Heinrich u. Lieberich, Heinz: Deutsche Rechtsgeschichte, ${ }^{19} 1992$.

Moнnhaupt, Heinz: Rechtsgeschichte in den beiden deutschen Staaten (1988-1990). Beispiele, Parallelen, Positionen, 1991.

OGRIs, Werner: Die Rechtsentwicklung in Österreich 1848-1918, 1975.

Planitz, Hans: Grundzüge des deutschen Privatrechts, ${ }^{3} 1949$.

Planitz, Hans u. Eckhardt, Karl August: Deutsche Rechtsgeschichte, ${ }^{3} 1971$.

Putrkamer, Ellinor von $(\mathrm{Hg}$.): Föderative Elemente im deutschen Staatsrecht seit 1648, 1955 = Quellensammlung zur Kulturgeschichte 7 .

RADBRUCH, Gustav: Vorschule der Rechtsphilosophie, ${ }^{3} 1965=\mathrm{Kl}$. Vandenhoeck-Reihe 80/81 (auch in: RADBRUCH, Gustav: Gesamtausgabe, hg. v. Arthur Kaufmann, Bd. 3: Rechtsphilosophie III, bearb. v. Winfried Hassemer, $1990,121-227)$. 
Rechtshistorisches Journal (RJ), $1982 \mathrm{ff}$.

ReIBSTEIN, Ernst: Völkerrecht. Eine Geschichte seiner Ideen in Lehre und Praxis, 2 Bde., 1958, 1963.

Rivista Internazionale di Diritto Comune (RIDC), $1990 \mathrm{ff}$.

RUPING, Hinrich: Grundriß der Strafrechtsgeschichte, ${ }^{2} 1991$.

SEAGLE, William: Weltgeschichte des Rechts, ${ }^{3} 1967$.

SCHLosser, Hans: Grundzüge der Neueren Privatrechtsgeschichte. Ein Studienbuch, ${ }^{7} 1993=$ UTB 882 .

ScнміDт, Eberhard: Einführung in die Geschichte der deutschen Strafrechtspflege, ${ }^{3} 1965$ (Nachdr. 1995).

Schotr, Clausdieter: Rechtsgeschichte. Texte und Lösungen, ${ }^{4} 1992$.

SCHRÖDER, Richard u. KUnsSBERG, Eberhard Frhr. von: Lehrbuch der deutschen Rechtsgeschichte, '1932 (Nachdr. 1966).

Schulze, Hans K.: Grundstrukturen der Verfassung im Mittelalter, 2 Bde., ${ }^{3} 1995,{ }^{2} 1992=$ Urban Tb. 371/372.

Schulze, Reiner: Europäische Rechts- und Verfassungsgeschichte. Ergebnisse und Perspektiven der Forschung, 1991.

Schwerin, Claudius Frhr. von u. Thieme, Hans: Grundzüge der deutschen Rechtsgeschichte, ${ }^{4} 1950$.

Sellert, Wolfgang, NeEF, Friedhelm u. RUPING, Hinrich: Studien- und Quellenbuch zur Geschichte der deutschen Strafrechtspflege, 2 Bde., 1989, 1994.

SPRANDEL, Rolf: Verfassung und Gesellschaft im Mittelalter, ${ }^{5} 1994=$ UTB 461.

Stintzing, Roderich von u. Landsberg, Ernst: Geschichte der Deutschen Rechtswissenschaft, 3 Abteilungen in 4 Bdn., 1880-1910 (Neudr. 1957).

Sтовве, Otto: Geschichte der deutschen Rechtsquellen, 2 Bde., 1860, 1864.

STOLLEIS, Michael: Geschichte des öffentlichen Rechts in Deutschland, 2 Bde., 1988, 1992.

Stolleis, Michael (Hg.): Juristen. Ein biographisches Lexikon. Von der Antike bis zum 20. Jahrhundert, 1995.

Tijdschrift voor Rechtsgeschiedenis (TRG), $1918 \mathrm{ff}$.

VERDROss, Alfred: Abendländische Rechtsphilosophie. Ihre Grundlagen und Hauptprobleme in geschichtlicher Schau, ${ }^{2} 1963$.

Vormbaum, Thomas ( $\mathrm{Hg}$.): Texte zur Strafrechtstheorie der Neuzeit, 2 Bde., 1993.

WESEL, Uwe: Juristische Weltkunde, ${ }^{7} 1994$ = stw 467.

Wesenberg, Gerhard u. Wesener, Gunter: Neuere deutsche Privatrechtsgeschichte im Rahmen der europäischen Rechtsentwicklung, ${ }^{4} 1985$.

WIEACKER, Franz: Privatrechtsgeschichte der Neuzeit unter besonderer Berücksichtigung der deutschen Entwicklung, ${ }^{2} 1967$ (Nachdr. 1996).

Willowerr, Dietmar: Deutsche Verfassungsgeschichte. Vom Frankenreich bis zur Teilung Deutschlands, ${ }^{2} 1992$.

Wolf, Erik (Hg.): Quellenbuch zur Geschichte der deutschen Rechtswissenschaft, 1950.

Wolf, Erik: Große Rechtsdenker der deutschen Geistesgeschichte, ${ }^{4} 1963$. 


\section{Ausgewählte Studienliteratur}

Zeitschrift der Savigny-Stiftung für Rechtsgeschichte (ZRG): Germanistische Abteilung (GA), Romanistische Abteilung (RA), 1880 ff., Kanonistische Abteilung (KA), $1911 \mathrm{ff}$.

Zeitschrift für Neuere Rechtsgeschichte (ZNR), 1979 ff.

Zeumer, Karl (Hg.): Quellensammlung zur Geschichte der Deutschen Reichsverfassung in Mittelalter und Neuzeit, ${ }^{2} 1913$ (Nachdr. 1987).

ZIEGLER, Karlheinz: Völkerrechtsgeschichte, 1994.

Zippelius, Reinhold: Kleine deutsche Verfassungsgeschichte. Vom frühen Mittelalter bis zur Gegenwart, ${ }^{2} 1995$. 
